\title{
Evaluation of theileria parva enolase as a target for designing new generation antiheilerial drug
}

\author{
Irmak Icen Taskin ${ }^{\mathrm{a},}$ *, Omer Munzuroglu ${ }^{\mathrm{b}}$ \\ a Firat University, Department of Biology, TR-23119, Elazig Turkey \\ b Firat University, Department of Bioengineering, TR-23119, Elazig Turkey
}

\section{ART I C LE IN FO}

\section{Article history:}

Received 14 March 2017

Received in revised form 03May 2017

Accepted 03 May 2017

\section{Keywords:}

Theileria parva,

Enolase,

Anti-theilerial drugs,

Homology modeling.

\begin{abstract}
A B S T R A C T
Enolase (2-phospho-D-glycerate hydrolase) enzyme has been characterized extensively and approved as a molecular drug target for rational drug discovery in apicomplexan such as Plasmodium spp., Toxoplasma gondii but not in the Theileria parva which causative agent of East Coast Fever engender serious economic losses. For the aim of identifying a key drug target, we have analyzed Theileria parva's enolase sequence and we have constructed three-dimensional structure of that enolase and mammalian host Bos taurus enolase (BtEno). Constructed model was approved, structurally analyzed and conceivable ligand binding pockets were identified for the first time in the literature. In this study, we reported significant findings, a pentapeptide and three different dipeptide insertions in TpEno ( $\mathrm{D}_{103} \mathrm{~W}_{104} \mathrm{G}_{105} \mathrm{Y}_{106} \mathrm{C}_{107}, \mathrm{~T}_{147} \mathrm{D}_{148}, \mathrm{~K}_{261} \mathrm{E}_{262}, \mathrm{~K}_{317} \mathrm{~L}_{318}$ ), when compare the sequences of enolase gene with its counterpart host BtEno. The homology modeling revealed that these insertions constituted loops that absent in B. taurus enolase seem to be important lead structures which can be used as binding sites for inhibition of $T p$ Eno.
\end{abstract}

\section{Introduction}

Apicomplexan parasites cause destructive human and animal diseases, which lead to the remarkable amount of economic losses throughout the world (Kumpula and Kursula, 2015). The most widely known examples of those parasites are Plasmodium spp., Toxoplasma gondii, Theileria parva and Theileria annulata, which cause phylum-caused malaria, toxoplasmosis and east coast fever and tropical theileriosis, respectively. The East Coast Fever (ECF), which is also known as corridor disease, is an important tick-borne disease of cattle and it causes approximately $\$ 200$ million of loss for farmers in Africa annually (Olwoch et al., 2008).

The actual approaches for preventing these diseases caused by Theileria spp. reside on the utilization of acaricides for the tick control (Walker, 2007), and chemotherapy (McHardy et al.,1985; Dolan et al., 1992) or immunization via the live attenuated cell-line vaccine for controlling the parasite in endemic areas (Bishop et al., 2001). But, there are also some factors limiting the efficiency of struggle with disease such as high costs and resistance to acaricides (Walker, 2007), requirement of liquid-nitrogen cold-chain for transferring the vaccine, possibility of vaccine-induced carrier stage, and also infection risk for unvaccinated cattle that graze together with vaccinated ones (Oura et al., 2004). When used in early period, parvaquone and buparvaquone (Mchardy et al., 1985; Dolan et al., 1992) are the unique efficient chemotherapy options for parasitic development, because extensive destruction of hematopoietic and lymphoid tissues occurs in the later stage of the disease (Singh et al., 1993) . However, recently, it was reported that resistance had developed against the antitheilerial. The first report on buparvaquone resistance has been published in the year 2010 (Mhadhbi et al., 2010) and followed by a new case in the year 2012 caused by the point mutation mitochondrial cytochrome $b$ gene in parasite (Sharifiyazdi et al.,2012; Mhadhbi et al., 2015). As a result of these studies, it has been understood that there is a necessity for immediately developing a novel antitheilerial medication

\footnotetext{
* Corresponding author. 
that acts through different modes of action.

An important chemotherapeutic target is the glycolytic pathway of parasitic protozoa since it is the only way of meeting their energy requirements. After the genome sequencing of T. parva has been accomplished in year 2005 (Gardner et al., 2005), it has been achieved important information about the metabolism of this parasite (Hayashida et al., 2013; Lau, 2009). The result signified Theileria spp. have an unusual TCA cycle as only three of the four pyruvate dehydrogenase complex (PDH) subunits have been identified (Gardner et al., 2005). Therefore, it has been suggested that $T$. parva, like Plasmodium spp., doesn't have any conventional TCA-cycle and dependence on glycolysis as a source of energy (Lau, 2009).

Enolase (2-phospho-D-glycerate hydrolase) is a necessary dimeric glycolytic enzyme, which acts as a catalyzer of interconversion of 2-phosphoglyceric acid (2-PGA) and phosphoenolpyruvate (PEP) during the glycolysis process (Labbe et al., 2006). That's why; the gene that is known to encode the T. parva enolase (TpEno) was chosen as the drug target since it is the main enzyme throughout the glycolytic process. In present study sequence analysis of $T p$ Eno and the homology modeling for the 3D structure of TpEno were performed for the first time in the literature. The aim of this process is to determine the key drug target through the comparative analyses of the 3D structure of the target and the mammalian host Bos taurus enolase (BtEno).

\section{Materials and methods}

\subsection{Alignment of Amino Acid Sequence of TpEno with Some Known Enolase Sequences}

Sequence of the amino acid belong to enolase of T. parva (TpEno), other apicomplexan parasites, host B. taurus and plants were retrieved from the National Centre for Biotechnology Information database (NCBI). The NCBI reference sequence used in this study were XP_764336.1, AAK38886.1, AAG60329.1, AAA18634.1, NP_001029874.1, AAM12985.1 for Theileria parva, Eimeria tenella, Toxoplasma gondii, Plasmodium falciparum, Bos taurus and Arabidopsis thaliana respectively. Lipper Centre for Computational Genetics, Harvard were used to comparing reverse and forward strand sequences. Sequences alignments at amino acid level were achieved by utilizing ClustalW2 (Chenna et al., 2003). The positions of exons and introns were determined by the GSDS utility (Guo et al., 2007).

\subsection{Physiochemical, Functional Properties and Secondary Structure Prediction of TpEno}

The physiochemical properties of TpEno, such as theoretical isoelectric point $(\mathrm{pI})$, molecular weight, the total number of positive and negative residues, extinction coefficient, instability index, aliphatic index and grand average hydropathy (GRAVY), were performed by Expasy's ProtParam server (Gasteiger et al., 2003). Secondary structure properties of the TpEno sequences was calculated by web-based program SOPMA (Geourjon and Deleage, 1995).

\subsection{Prediction and validation $3 D$ structure of the TpEno and BtEno}

An automatic 3D model of TpEno and BtEno were constructed based on structural homologies with a template protein using the program Protein Homology/analogy Recognition engine 2 (PHYRE2) a free online homology modeling server (Kelley et al., 2015). This program utilizes the alignment of hidden Markov models through HHsearch to remarkably increase the efficiency of alignment and recognition rate. Energy minimization and root mean square deviation (RMSD) were performed using 3Drefine (Bhattacharya and Cheng, 2013) and SuperPose servers (Bhattacharya and Cheng, 2013), respectively. To ensure the quality of modeling, stereochemistry, energy profile and residue environment of modeled structure were checked by different servers. RAMPAGE (Lovell et al., 2003) was used to test the stereochemistry of the model. The model was further examined by ProSA (Wiederstein and Sippl, 2007) and ERRAT servers (Colovos and Yeates, 1993). Protein Structure Validation Suite-PSVS 1.3 (http://psvs-1_4-dev.nesg.org) was used to analyses the quality of the model.

\subsection{Ligand Binding Site Identification}

MetaPocket 2.0 server (Huang, 2009) was used to determine possible ligand-binding sites. The MetaPocket is an accord strategy enhanced at Technical University of Dresden and Zhejiang University together, in which the predicted binding sites from eight techniques, for example, PASS11 (PAS), LigsiteCS (LCS), Q_SiteFinder (QSF), GHECOM (GHE), POCASA (PCS), Fpocket (FPK), SURFNET (SFN), ConCavity (CON) are consolidated to build up the forecast achievement rate.

\section{Result}

\subsection{Alignment of Amino Acid Sequence of TpEno with Some Known Enolase Sequences}

The amino acid sequence of TpEno (XP_764336.1) was aligned with the amino acid sequences of some other species, Bos Taurus (NP_001029874.1), Plasmodium falciparum (AAA18634.1), Toxoplasma gondii (AAG60329.1), Eimeria tenella (AAK38886.1) and Arabidopsis thaliana (AAM12985.1) by ClustalW software. Alignment result revealed the presence of insertions or deletions at the same positions of TpEno compared of host organism Bos taurus (Fig. 1). The most striking insertion consisted in the addition of a pentapeptide, three dipeptide insertions in TpEno, $\mathrm{D}_{103} \mathrm{~W}_{104} \mathrm{G}_{105} \mathrm{Y}_{106} \mathrm{C}_{107}$, $\mathrm{T}_{147} \mathrm{D}_{148}, \mathrm{~K}_{261} \mathrm{E}_{262}, \mathrm{~K}_{317} \mathrm{~L}_{318}$ respectively absent in BtEno (shown with a red frame at Fig. 1). This a pentapeptide and three dipeptide residue insertion may be a valuable unique target for antitheilerial design. Our alignment result also demonstrated that other apicomplexan parasites had similar insertion sites like TpEno (Fig. 1).

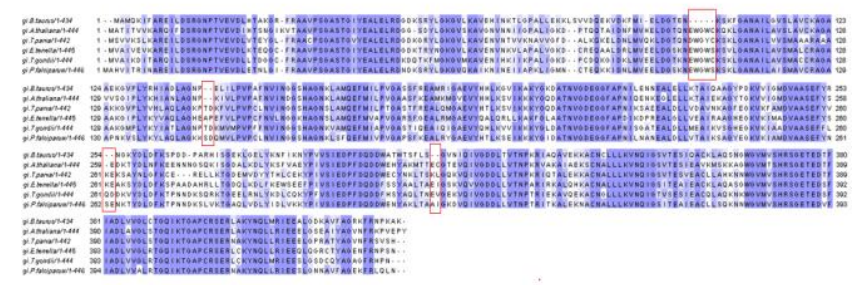

Figure 1. Alignment of TpEno amino acid sequence (XP_764336.1) with Enolase from Bos taurus (NP_001029874.1) Plasmodium falciparum (AAA18634.1), Toxoplasma gondii (AAG60329.1), Eimeria tenella (AAK38886.1) and Arabidopsis thaliana (AAM12985.1). The five and two residue insertions in apicomplexan parasite enolase are shown in the box. 


\subsection{Physiochemical, Functional Properties and Secondary Structure Prediction of TpEno}

Expasy's ProtParam server used to check stereochemistry, energy profiles, potential errors, nonbonding interactions and some physical and chemical properties of TpEno. Protein model is about $48056.01 \mathrm{kDa}$ and calculated pI (isoelectric point) of the $T p$ Eno is 6.43 . The total number of positively charged residues (Arg+Lys) is 53\% and negatively charged residues $(\mathrm{Asp}+\mathrm{Glu})$ is $55 \%$. The instability index of this enzyme was calculated as 27.68 which classifies the protein as stable. The aliphatic index (AI) which is defined as the relative volume of a protein occupied by aliphatic side chains ( $A, V, I$ and $L)$, is regarded as a positive factor for the increase in thermal stability of globular proteins, and the calculated value was found to be 90.23. The grand average of hydropathicity (GRAVY) value is -0.186 . The Secondary structure of TpEno was predicted by the self-optimized prediction method with the alignment (SOPMA) server. According to the result, alpha helix $(45.70 \%)$ and random coil $(27.83 \%)$ compose the large proportion of the physical feature. Extended strands and beta turns have found as $16.52 \%$ and $9.95 \%$ respectively.

\subsection{Homology Modelling, Validation and Structural Differences between Enolase of T. parva and B. taurus}

Based on the investigation in Plasmodium species, we investigated to determine a key drug target in view of homology modeling to design a highly specific drug based on the homology modeling three-dimensional (3D) structure of $T p$ Eno as a target with the final aim considering of data get from the sequence of TpEno. The amino acid sequence obtained from the NCBI (NCBI Reference Sequence: XP_764336.1) was used to model the 3D structure of TpEno (Figure 2. A) and $B$. taurus enolase (NCBI Reference Sequence: NP_001029874.1) (Figure 2. B) by Phyre2 workspace for comparison with TpEno. Templates used for homology modeling of TpEno PDB ID was 30TR and 2AKM was for BtEno.
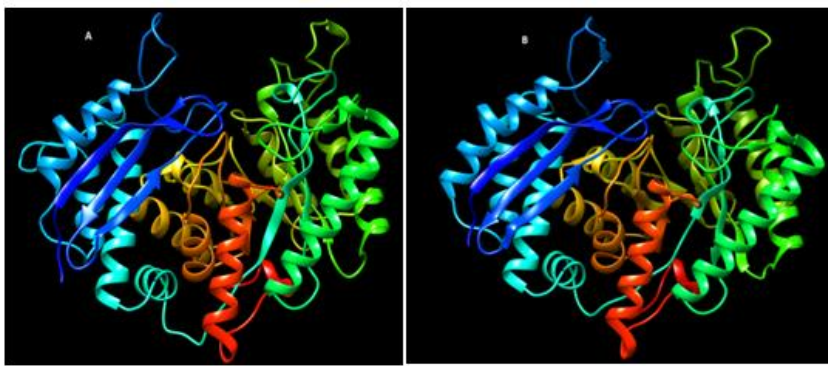

Figure 2. 3D structures of the enolase enzymes predicted through homology modeling: A-3D structures of enolase protein of T. parva generated by Phyre2 B-3D structures of enolase protein of mammalian host $B$. taurus generated by Phyre2.

The Ramachandran plot for the predicted model by RAMPAGE reveals that $95.7 \%$ (421) residues were found in the most favorable region, $3.2 \%$ (14) residues in allowed regions and $1.1 \%$ (5) residues in outlier region (Fig. 3).

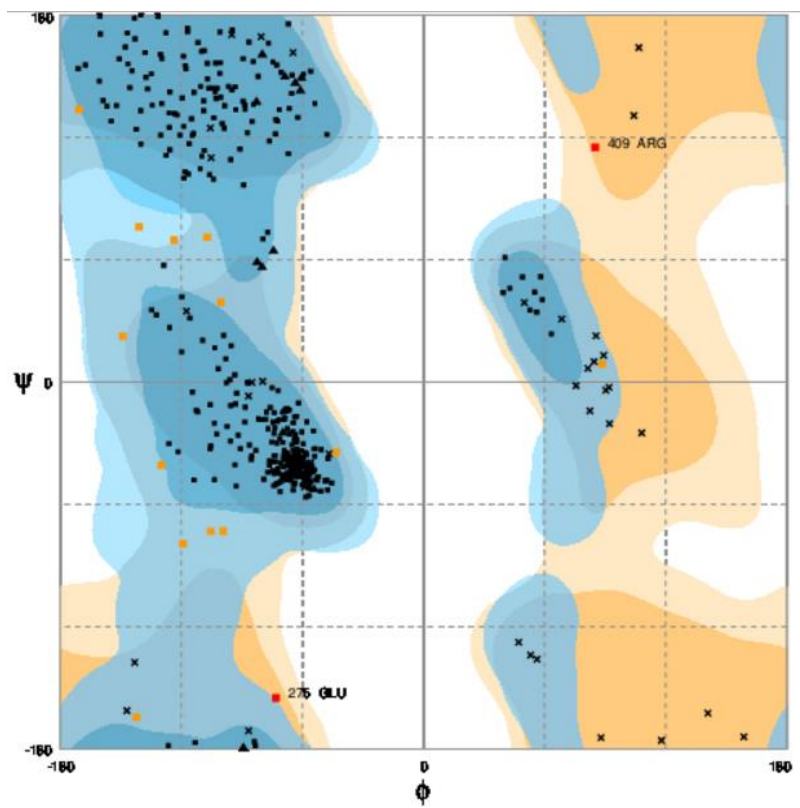

Figure 3. Ramachandran plot of Enolase from Theileria parva obtained by RAMPAGE: $95.7 \%$ (421) residues were found in most favorable region, $3.2 \%(14)$ residues in allowed regions and $1.1 \%(5)$ residues in outlier region.

For predict non-bonding interactions by examining the statistics of pairwise atomic interaction, ERRAT served was used. The obtained result showed that the model has a good resolution structure with 90.092 overall quality factor. ProSA server used for assessment overall quality of the modeled protein via the scores of all experimentally determined protein chains currently available in the Protein Data Bank (PDB. The program gave a-9.85 z-score and a plot of the residue energies for TpEno demonstrates modeled quality is acceptable and protein in model was within the range of the typically found proteins of similar size (Fig. 4).
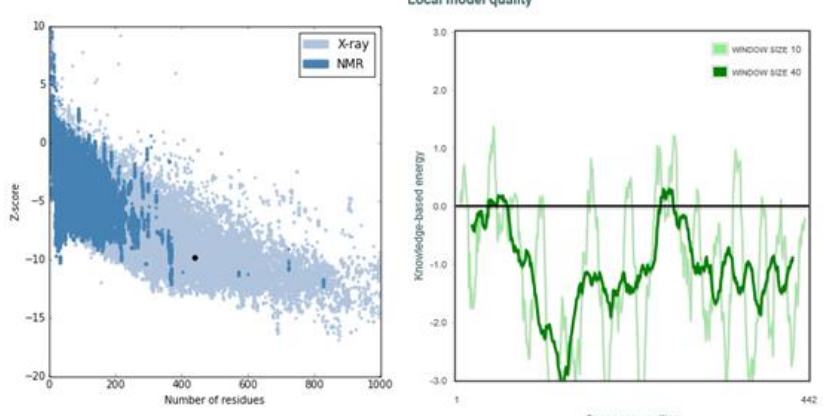

Figure 4. ProSA web service analysis of $T p$ Eno protein model.

Structural differences between homology modeling were showed by overlaying 3D structures of T. parva and Bos taurus enolase. Formation of an insertion of five amino acids (D ${ }_{103} \mathrm{~W}_{104} \mathrm{G}_{105} \mathrm{Y}_{106} \mathrm{C}_{107}$ ) (Figure 5. A) and three dipeptide insersitions are $\mathrm{T}_{147} \mathrm{D}_{148}, \mathrm{~K}_{261} \mathrm{E}_{262}, \mathrm{~K}_{317} \mathrm{~L}_{318}$ ) (Figure 5. B, C, and $\mathrm{D}$ respectively) clearly seen in $T p$ Eno overlayed to its mammalian host B. taurus. 


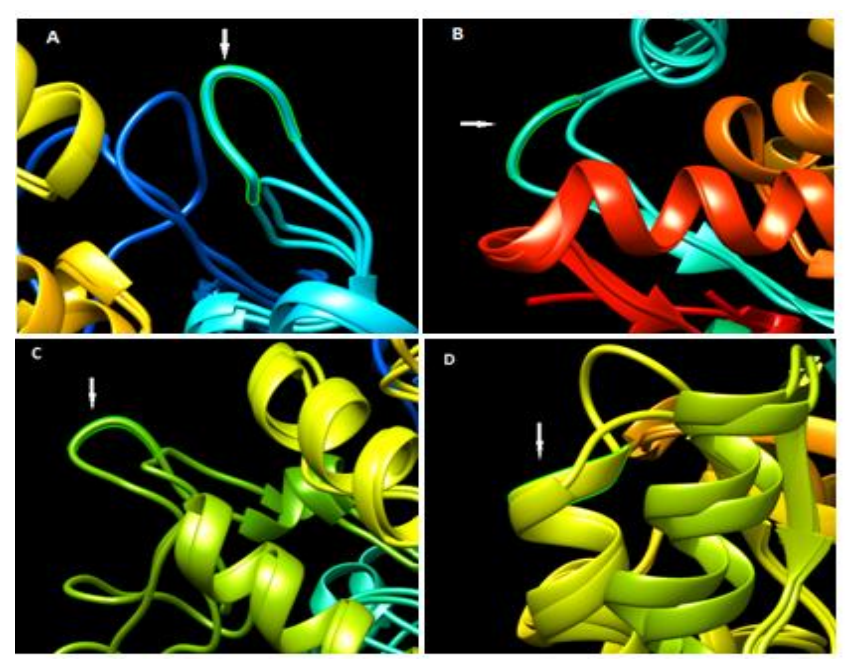

Figure 5. Three dimensional superposition of T. parva enolase and $B$. taurus enolase, A-The pentapeptide insertion $\mathrm{D}_{103} \mathrm{~W}_{104} \mathrm{G}_{105} \mathrm{Y}_{106} \mathrm{C}_{107}$ $T p$ Eno is shown with white arrow, B-The dipeptide insertion $\left(\mathrm{T}_{147} \mathrm{D}_{148}\right)$ in $T p$ Eno is shown with the white arrow, $\mathbf{C}$-The dipeptide insertion $\left(\mathrm{K}_{261} \mathrm{E}_{262}\right)$ in $T p$ Eno is with the white arrow, D-Another dipeptide insertion $\left(\mathrm{K}_{317} \mathrm{~L}_{318}\right)$ in $T p$ Eno is with the white arrow.

\subsection{Ligand Binding Site Identification}

Protein-ligand binding site estimation from a 3D protein structure plays an essential role in rational drug design and can be helpful in drug side-effects prediction or clarification of protein function. In this study, we used MetaPocket 2.0 with considering top three sites to give the notice ligand binding side of $T p$ Eno which structure has not been solved yet (Table 1). After grouping the main 3 sites from PAS, LCS, FPK, SFN, GHE, CON, there are 7 metaPocket clusters. The first MetaPocket site (MPK1) include 4 pocket sites; the first one from LigisiteCS (LCS-1), the first one from PASS (PAS-1), the first one from GHECOM (GHE-2), the first one from Concavity (CON-1) with total $11.42 \mathrm{z}$ score. The second MetaPocket site (MPK2) contains 3 pocket sites; SFN-1(SURFNET), FPK1(Fpocket) and CON-3 with the total $10.16 \mathrm{z}$ score. The third MetaPocket site (MPK3) contains 4 pocket sites from PAS-2, SFN-2, LCS-3, GHE-1 and total $8.13 \mathrm{z}$ score. The fourth MetaPocket site contains 2 pocket sites with $4.49 \mathrm{z}$ score from LCS-2, CON-2. The fifth MetaPocket site contains 3 pocket sites from FPK-3, PAS-3, GHE-3 with total 2.06 z-score. The sixth MetaPocket site contains 1 pocket sites from SFN-3 and total zscore is 1.06 . Table 1 shows the potential binding sites residue from modeled TpEno (Table. 1).

Table 1. Ligand binding side prediction of T.parva enolase: The potential 3 ligand binding sites of $T p$ Eno. The possible binding sites of 3 MetaPockets are shown and they are present in residue format with each line beginning with 'RESI'. The residue specification in the table is assembled of three parts: residue name, chain indicator and residue sequence number.

\begin{tabular}{|c|c|c|c|}
\hline $\begin{array}{l}\text { Header } \\
\text { Binding Site } 1\end{array}$ & & & \\
\hline $\begin{array}{l}\text { RESI GLU_9^174^ } \\
\text { LYS_9 } 9^{\wedge} 352^{\wedge}\end{array}$ & GLU_9^ $217^{\wedge}$ & $\mathrm{ASP}_{-} 9^{\wedge} 252^{\wedge}$ & ASP_9^ $327^{\wedge}$ \\
\hline $\begin{array}{l}\text { RESI SER_9^379^ } \\
\text { SER_9^ } 9^{\wedge} 382^{\wedge}\end{array}$ & HIS_9^ $9^{\wedge} 380^{\wedge}$ & LYS_9^ ${ }^{\wedge} 403^{\wedge}$ & ARG_9^381^ \\
\hline $\begin{array}{l}\text { RESI GLN_9^173^ } \\
\text { GLY_9^39^ } 39^{\wedge}\end{array}$ & HIS_9^165^ & ASP_9^328^ & ALA_9^ $40^{\wedge}$ \\
\hline 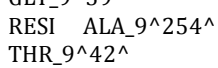 & SER_9^ $9^{\wedge} 41^{\wedge}$ & ASP_9^301^ & GLU_9^ $257^{\wedge}$ \\
\hline
\end{tabular}

\begin{tabular}{|c|c|c|c|}
\hline $\begin{array}{l}\text { RESI GLU_9^ } 46^{\wedge} \\
\text { ASP_9^} 216^{\wedge} \\
\text { RESI GLU_9^300^ }\end{array}$ & 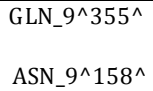 & 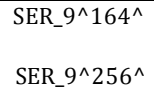 & 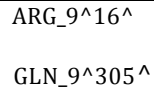 \\
\hline \multicolumn{4}{|l|}{$\begin{array}{l}\text { Header } \\
\text { Binding Site } 2\end{array}$} \\
\hline 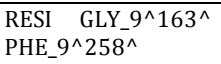 & LEU_9^170^ & ALA_9^171^ & MET_9^172^ \\
\hline 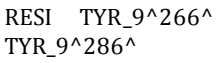 & SER_9^ $226^{\wedge}$ & ALA_9^227^ & GLU_9^ $228^{\wedge}$ \\
\hline $\begin{array}{l}\text { RESI LEU_9^ } 290^{\wedge} \\
\text { LEU_9^} 276^{\wedge}\end{array}$ & MET_9^283^ & HIS_9^ $289^{\wedge}$ & ASP_9^ $285^{\wedge}$ \\
\hline 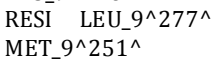 & LYS_9^278^ & ASN_9^ $267^{\wedge}$ & GLU_9^275^ \\
\hline 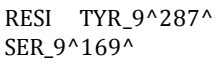 & ILE_9^296^ & ILE_9^299^ & LYS_9^ $225^{\wedge}$ \\
\hline $\begin{array}{l}\text { RESI PHE_9^} 270^{\wedge} \\
\text { CYS_9^ }^{\wedge} 291^{\wedge}\end{array}$ & GLU_9^ $273^{\wedge}$ & LYS_9^271^ & CYS_9^ $9^{\wedge} 272^{\wedge}$ \\
\hline $\begin{array}{l}\text { RESI LEU_9^318^ } \\
\text { SER_9^} 264^{\wedge}\end{array}$ & VAL_9 $9^{\wedge} 322^{\wedge}$ & LEU_9^314^^ & TYR_9^259^ \\
\hline $\begin{array}{l}\text { RESI THR_9^ }{ }^{\wedge} 29^{\wedge} \\
\text { ASP_9^} 307^{\wedge}\end{array}$ & GLY_9^ $280^{\wedge}$ & ASP_9^ $281^{\wedge}$ & PHE_9^ $303^{\wedge}$ \\
\hline $\begin{array}{l}\text { RESI CYS_9 } 9^{\wedge} 310^{\wedge} \\
\text { VAL_9^} 253^{\wedge} \\
\text { RESI SFR }\end{array}$ & ASP_9^ $304^{\wedge}$ & $\mathrm{PRO}_{-} 9^{\wedge} 302^{\wedge}$ & ALA_9^255^^ \\
\hline $\begin{array}{l}\text { Header } \\
\text { Binding Site } 3\end{array}$ & & & \\
\hline $\begin{array}{l}\text { RESI ARG_9^} 438^{\wedge} \\
\text { HIS_9^} 442^{\wedge}\end{array}$ & SER_9^439^ & HIS_9^ $370^{\wedge}$ & ASN_9^373^ \\
\hline 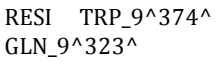 & SER_9^ $9^{\wedge} 41^{\wedge}$ & GLY_9^375^ & ASN_9^347^ \\
\hline $\begin{array}{l}\text { RESI LYS_9^ } 9^{\wedge} 371^{\wedge} \\
\text { GLU_9^} 342^{\wedge}\end{array}$ & ASN_9^372^ & LEU_9^341^ & LYS_9^344^ \\
\hline $\begin{array}{l}\text { RESI VAL_9^} 376^{\wedge} \\
\text { LYS_9^ } 343^{\wedge}\end{array}$ & CYS_9^346^ & GLY_9^319^ & THR_9^315^ \\
\hline RESI ASN_9^312^ & & & \\
\hline
\end{tabular}

\section{Conclusions}

The determination of macromolecular targets that are necessary for the life of parasite is the first action for the drug design. Glycolytic enzymes are considered to have potential from this aspect. The energy supply of Plasmodium spp. are based on the glycolytic process (Foth et al., 2005). As a result of extensive studies on Plasmodium spp. glycolytic enzymes, such as aldolase (Certa et al., 1988), lactate dehydrogenase (Cameron et al., 2004; Piper et al., 1999), enolase (PalBhowmick et al., 2004), and triose phosphate isomerase (Maithal et al., 2002) were shown to have potential as detection tools, drug targets and vaccine candidates . Plasmodium spp. are the most species subjected to the structure based drug design among apicomplexan parasites. The crystal structure of Plasmodium falciparum LDH $(P f L D H)$ reveals a significant property, a penta peptide insertion in the active site of the enzyme, leads to a separate cavity in the surface adjacent to the catalytic region that is mammalian equivalent LDH does not have the pentapeptide insertion (Dunn et al., 1996). Based on these data, azole based inhibitors developed to bind active side of the of PfLDH protein to prevent the enzyme activity and the parasite advancement in red platelets (Cameron et al., 2004). Among studies, it has been determined that the deletion of pentapeptide insertion (EWGWS) from the active region of $P$. falciparum enolase caused the decrease of kcat/Km values for 100 times (Pal-Bhowmick et al., 2004). The mice vaccinated with $P$. falciparum enolase modified by deletion of this pentapeptide insertion region showed a trivial reaction when compared wild-type enolase. It has also been demonstrated 
that this insertion region is a necessity for the proper operation of enolase and it may generate a conservative antigenic epitope in parasite's enolase (Pal-Bhowmick et al., 2004). From this aspect, specific inhibitors may be developed through cloning the parasitic enolase and comparatively analyzing with host mammalian enolase. In order to design a structure-based medication, we chose the Theileria parva enolase as our target. 3D structure was constructed and analyzed in combination with homology modeling and possible ligand binding sites have been identified in our study. As an important finding, a pentapeptide and three dipeptide insertions in TpEno ( $\mathrm{D}_{103} \mathrm{~W}_{104} \mathrm{G}_{105} \mathrm{Y}_{106} \mathrm{C}_{107}, \mathrm{~T}_{147} \mathrm{D}_{148}, \mathrm{~K}_{261} \mathrm{E}_{262}, \mathrm{~K}_{317} \mathrm{~L}_{318}$ ) were defined through comparing to the homology model of TpEno and its counterpart host BtEno. It was determined that the insertion regions, which are present on T.parva enolase, absent in host B. taurus enolase. Hence, these insertion sites can be used as the binding sites for specific enzyme inhibitors to be developed.

\section{References}

Bhattacharya, D., and Cheng, J., 2013. 3Drefine: consistent protein structure refinement by optimizing hydrogen bonding network and atomic-level energy minimization. Proteins, 81, 119-131.

Bishop, R., Geysen, D., Spooner, P., Skilton, R., Nene, V., Dolan, T., and Morzaria, S., 2001. Molecular and immunological characterisation of Theileria parva stocks which are components of the 'Muguga cocktail' used for vaccination against East Coast fever in cattle. Vet Parasitol, 94, 227-237.

Cameron, A., Read, J., Tranter, R., Winter, V. J., Sessions, R. B., Brady, R. L., Vivas, L., Easton, A., Kendrick, H., Croft, S. L., Barros, D., Lavandera, J. L., Martin, J. J., Risco, F., Garcia-Ochoa, S., Gamo, F. J., Sanz, L., Leon, L., Ruiz, J. R., Gabarro, R., Mallo, A., and Gomez de las Heras, F. , 2004. Identification and activity of a series of azole-based compounds with lactate dehydrogenase-directed anti-malarial activity. J Biol Chem, 279, 31429-31439.

Certa, U., Ghersa, P., Dobeli, H., Matile, H., Kocher, H. P., Shrivastava, I. K., Shaw, A. R., and Perrin, L. H., 1988. Aldolase activity of a Plasmodium falciparum protein with protective properties. Science, 240, 1036-1038.

Chenna, R., Sugawara, H., Koike, T., Lopez, R., Gibson, T. J., Higgins, D. G., and Thompson, J. D., 2003. Multiple sequence alignment with the Clustal series of programs. Nucleic Acids Res, 31, 3497-3500.

Colovos, C., and Yeates, T. O. , 1993. Verification of protein structures: patterns of nonbonded atomic interactions. Protein Sci, 2, 1511-1519.

Dolan, T. T., Injairu, R., Gisemba, F., Maina, J. N., Mbadi, G., Mbwiria, S. K., Mulela, G. H., and Othieno, D. A., 1992. A clinical trial of buparvaquone in the treatment of East Coast fever. Vet Rec, 130, 536538.

Dunn, C. R., Banfield, M. J., Barker, J. J., Higham, C. W., Moreton, K. M., Turgut-Balik, D., Brady, R. L., and Holbrook, J. J. , 1996. The structure of lactate dehydrogenase from Plasmodium falciparum reveals a new target for anti-malarial design. Nature Structural Biology, 3, 912-915.

Foth, B. J., Stimmler, L. M., Handman, E., Crabb, B. S., Hodder, A. N., and McFadden, G. I., 2005. The malaria parasite Plasmodium falciparum has only one pyruvate dehydrogenase complex, which is located in the apicoplast. Molecular Microbiology, 55, 39-53.

Gardner, M. J., Bishop, R., Shah, T., de Villiers, E. P., Carlton, J. M., Hall, N., Ren, Q., Paulsen, I. T., Pain, A., Berriman, M., Wilson, R. J., Sato, S., Ralph, S. A., Mann, D. J., Xiong, Z., Shallom, S. J., Weidman, J., Jiang, L., Lynn, J., Weaver, B., Shoaibi, A., Domingo, A. R., Wasawo, D., Crabtree, J., Wortman, J. R., Haas, B., Angiuoli, S. V., Creasy, T. H., Lu, C., Suh, B., Silva, J. C., Utterback, T. R., Feldblyum, T. V., Pertea, M., Allen, J., Nierman, W. C., Taracha, E. L., Salzberg, S. L., White, O. R., Fitzhugh, H.
A., Morzaria, S., Venter, J. C., Fraser, C. M., and Nene, V., 2005. Genome sequence of Theileria parva, a bovine pathogen that transforms lymphocytes. Science, 309, 134-137.

Gasteiger, E., Gattiker, A., Hoogland, C., Ivanyi, I., Appel, RD, Bairoch A., Gardner, M. J., Bishop, R., Shah, T., de Villiers, E. P., Carlton, J. M., Hall, N., Ren, Q., Paulsen, I. T., Pain, A., Berriman, M., Wilson, R. J., Sato, S., Ralph, S. A., Mann, D. J., Xiong, Z., Shallom, S. J., Weidman, J., Jiang, L., Lynn, J., Weaver, B., Shoaibi, A., Domingo, A. R., Wasawo, D., Crabtree, J., Wortman, J. R., Haas, B., Angiuoli, S. V., Creasy, T. H., Lu, C., Suh, B., Silva, J. C., Utterback, T. R., Feldblyum, T. V., Pertea, M., Allen, J., Nierman, W. C., Taracha, E. L., Salzberg, S. L., White, O. R., Fitzhugh, H. A., Morzaria, S., Venter, J. C., Fraser, C. M., and Nene, V., 2005. Genome sequence of Theileria parva, a bovine pathogen that transforms lymphocytes. Science, 309(5731), 134-1372003 ExPASy: The proteomics server for in-depth protein knowledge and analysis. Nucleic Acids Res.31, 3784-8.

Geourjon, C, and Deleage G., 1995. SOPMA: significant improvements in protein secondary structure prediction by consensus prediction from multiple alignments. Comput Appl Biosci. 11, 681-4.

Guo, A. Y., Zhu, Q. H., Chen, X., and Luo, J. C., 2007. [GSDS: a gene structure display server]. Yi Chuan, 29, 1023-1026.

Hayashida, K., Abe, T., Weir, W., Nakao, R., Ito, K., Kajino, K., Suzuki, Y., Jongejan, F., Geysen, D., and Sugimoto, C., 2013. Whole-genome sequencing of Theileria parva strains provides insight into parasite migration and diversification in the African continent. DNA Res, 20, 209-220.

Huang, B., 2009. MetaPocket: a meta approach to improve protein ligand binding site prediction. Omics. 13, 325-30.

Kelley, L. A., Mezulis, S., Yates, C. M., Wass, M. N., and Sternberg, M. J., 2015. The Phyre2 web portal for protein modeling, prediction and analysis. Nat Protoc, 10, 845-858.

Kumpula, E. P., and Kursula, I., 2015. Towards a molecular understanding of the apicomplexan actin motor: on a road to novel targets for malaria remedies? Acta Crystallographica Section FStructural Biology Communications, 71, 500-513.

Labbe, M., Peroval, M., Bourdieu, C., Girard-Misguich, F., and Pery, P., 2006. Eimeria tenella enolase and pyruvate kinase: a likely role in glycolysis and in others functions. Int J Parasitol, 36, 1443-1452.

Lau, A. O., 2009. An overview of the Babesia, Plasmodium and Theileria genomes: a comparative perspective. Mol Biochem Parasitol, 164, 1-8.

Lovell, S. C., Davis, I. W., Arendall, W. B., 3rd, de Bakker, P. I., Word, J. M., Prisant, M. G., Richardson, J. S., and Richardson, D. C., 2003. Structure validation by Calpha geometry: phi,psi and Cbeta deviation. Proteins, $50,437-450$.

Maithal, K., Ravindra, G., Balaram, H., and Balaram, P., 2002. Inhibition of plasmodium falciparum triose-phosphate isomerase by chemical modification of an interface cysteine. Electrospray ionization mass spectrometric analysis of differential cysteine reactivities. J Biol Chem, 277, 25106-25114.

McHardy, N., Wekesa, L. S., Hudson, A. T., and Randall, A. W., 1985. Antitheilerial activity of BW720C (buparvaquone): a comparison with parvaquone. Res Vet Sci, 39, 29-33.

Mhadhbi, M., Chaouch, M., Ajroud, K., Darghouth, M. A., and BenAbderrazak, S., 2015. Sequence Polymorphism of Cytochrome b Gene in Theileria annulata Tunisian Isolates and Its Association with Buparvaquone Treatment Failure. PLoS One, 10, e0129678.

Mhadhbi, M., Naouach, A., Boumiza, A., Chaabani, M. F., BenAbderazzak, S., and Darghouth, M. A., 2010. In vivo evidence for the resistance of Theileria annulata to buparvaquone. Vet Parasitol, 169, 241-247.

Olwoch, J. M., Reyers, B., Engelbrecht, F. A., and Erasmus, B. F. N., 2008. Climate change and the tick-borne disease, Theileriosis (East Coast fever) in sub-Saharan Africa. Journal of Arid Environments, 72, 108-120. 
Oura, C. A., Bishop, R., Wampande, E. M., Lubega, G. W., and Tait, A. , 2004. The persistence of component Theileria parva stocks in cattle immunized with the 'Muguga cocktail' live vaccine against East Coast fever in Uganda. Parasitology, 129, 27-42.

Pal-Bhowmick, I., Sadagopan, K., Vora, H. K., Sehgal, A., Sharma, S., and Jarori, G. K., 2004. Cloning, over-expression, purification and characterization of Plasmodium falciparum enolase. Eur J Biochem, $271,4845-4854$

Piper, R., Lebras, J., Wentworth, L., Hunt-Cooke, A., Houze, S., Chiodini, P., and Makler, M., 1999. Immunocapture diagnostic assays for malaria using Plasmodium lactate dehydrogenase (pLDH). Am J Trop Med Hyg, 60, 109-118.

Sharifiyazdi, H., Namazi, F., Oryan, A., Shahriari, R., and Razavi, M., 2012. Point mutations in the Theileria annulata cytochrome $b$ gene is associated with buparvaquone treatment failure. Vet Parasitol, 187, 431-435.

Singh, D. K., Thakur, M., Raghav, P. R., and Varshney, B. C. , 1993. Chemotherapeutic trials with four drugs in crossbred calves experimentally infected with Theileria annulata. Res Vet Sci, 54, 68-71.

Walker, A. R., 2007. Theileriosis and the tick control conundrum: a better way forward? Vet J, 173, 248-249.

Wiederstein, M., and Sippl, M. J., 2007. ProSA-web: interactive web service for the recognition of errors in three-dimensional structures of proteins. Nucleic Acids Res, 35 (Web Server issue), W407-410. 